\title{
Emergence of Cooperation and Evolutionary Stability in Finite Populations
}

\section{Citation}

Nowak, Martin A., Akira Sasaki, Christine Taylor, and Drew Fudenberg. 2004. Emergence of cooperation and evolutionary stability in finite populations. Nature 428(6983): 646-650.

\section{Published Version}

http://dx.doi.org/10.1038/nature02414

\section{Permanent link}

http://nrs.harvard.edu/urn-3:HUL.InstRepos:3196331

\section{Terms of Use}

This article was downloaded from Harvard University's DASH repository, and is made available under the terms and conditions applicable to Other Posted Material, as set forth at http:// nrs.harvard.edu/urn-3:HUL.InstRepos:dash.current.terms-of-use\#LAA

\section{Share Your Story}

The Harvard community has made this article openly available.

Please share how this access benefits you. Submit a story.

\section{Accessibility}


Emergence of cooperation and evolutionary stability in finite populations

Martin A. Nowak, Akira Sasaki, Christine Taylor, Drew Fudenberg

Program for Evolutionary Dynamics, Harvard University, Cambridge MA 02138, USA

Department of Biology, Kyushu University, Fukuoka 812-8581, Japan

Department of Economics, Harvard University, Cambridge MA 02138, USA

The problem of cooperation ${ }^{1-8}$ is that defection is evolutionarily stable. If everybody in a population defects and one individual cooperates then this individual has a lower payoff and will be opposed by selection. Thus, the emergence of cooperation is thought to require specific mechanisms: for example, several cooperators have to arise simultaneously to overcome an invasion barrier ${ }^{9}$ or arise as spatial clusters ${ }^{10,11}$. This understanding is based on traditional concepts of evolutionary stability and dynamics of infinite populations ${ }^{12-16}$. Here we study evolutionary game dynamics in finite populations ${ }^{17-20}$ and show that a single cooperator using a reciprocal strategy ${ }^{3,21}$ can invade a population of defectors with a probability that corresponds to a net selective advantage. We specify the conditions for natural selection to favor the emergence of cooperation and derive conditions for evolutionary stability in finite populations.

Explaining the evolution of cooperation by natural selection has been a major theme of evolutionary biology since Darwin. The standard game dynamical formulation, which captures the essence of the problem, is the Prisoner's Dilemma. In the non-repeated game, defection dominates cooperation. In the repeated game, stratetegies like tit-for-tat (TFT) or win-stay, lose-shift allow cooperation, but the question is how do they arise in the first place? Always defect (AllD) is evolutionarily stable against invasion by TFT in traditional game dynamics of infinite populations.

Let us investigate a game between two strategies, $A$ and $B$, with payoff matrix

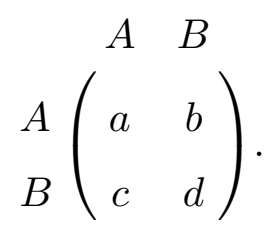


If $A$ and $B$ denote, respectively, TFT and AllD, then we normally have $a>c>d>b$. In this case, both TFT and AllD are strict Nash equilibria and evolutionarily stable strategies (ESS). Deterministic replicator dynamics of infinite populations admit an unstable equilibrium at a frequency of TFT given by $x^{*}=(d-b) /(a-b-c+d)$. If the initial frequency of TFT is less than this value, then it will be eliminated by natural selection. TFT can only replace AllD if its initial frequency exceeds this invasion barrier.

Let us now study a stochastic process describing a finite population of size $N$. At each time step, one individuals is chosen for reproduction proportional to fitness. The offspring replaces a randomly chosen individual. The population size is strictly constant ${ }^{22}$. The fitness of each player depends on the number of TFT or AllD players. In addition, we introduce a parameter $w$, which determines the contribution of the game's payoff to fitness. This parameter, quantifying the intensity of selection, cancels out in deterministic replicator dynamics of infinite populations, but plays a crucial role in finite populations, as we shall see.

We can calculate the probability, $\rho$, that starting from a single individual strategy $A$ will invade and take over a population of $B$ players (Methods). For a neutral mutant this fixation probability is $\rho=1 / N(\operatorname{Ref} 23)$. If $\rho>1 / N$ then selection favors $A$ replacing $B$. In Fig. 1, we show that in the case of TFT and AllD, $N \rho$ is a one-humped function of $N$. For a wide choice of parameter values, $a, b, c, d$ and $w$, there is an intermediate range of population sizes, $N$, with $N \rho>1$. Thus, the invasion and replacement of AllD by TFT, starting from a single indiviudal of TFT, can be favored by natural selection. Interestingly, there are critical minimum and maximum population sizes that allow positive selection of TFT: in very small populations, helping a competitor leads to a significant disadvantage; in very large populations, the selection against TFT at low frequencies is too strong. Thus, neither small nor large but intermediate population sizes are optimum for initiating cooperation.

Can we derive the underlying prinicple that determines whether a particular payoff matrix (1) allows selection for TFT replacing AllD? The exact expression for $\rho$ is complicated. The condition $\rho>1 / N$ requires the solution of $N$-th order polynomials, and a diffusion approximation yields transcendental equations. Nevertheless, the the following 
surprisingly simple theorem holds. For a given population size $N$ and sufficiently weak selection (small $w$ ), selection favors TFT replacing AllD if

$$
a(N-2)+b(2 N-1)>c(N+1)+d(2 N-4) .
$$

For the smallest possible population size, $N=2$ (it takes two to play), we obtain $b>c$. For the limit of large $N$, we obtain $a+2 b>c+2 d$. The latter condition is equivalent to $x^{*}<1 / 3$. Therefore, if the invasion barrier of TFT is less than $1 / 3$, there will be positive selection for TFT to replace AllD in a finite population.

In general, for any two strategies which are best replies to themselves, we find that selection can favor $A$ replacing $B$ for some $N$ and $w$, if $b>c$ or $x^{*}<1 / 3$ (Fig 2).

Our results have immediate consequences for the concept of evolutionary stability. The well-known definition of an evolutionarily stable strategy (ESS) is motivated by selection dynamics in infinite populations ${ }^{8}$. Strategy $B$ is ESS if either (i) $d>b$ or (ii) $d=b$ and $a<c$. These conditions imply that selection opposes the spread of infinitesimal small fractions of $A$ players in infinitely large populations of $B$.

For finite population size, $N$, we propose that $B$ is an evolutionarily stable strategy, $\mathrm{ESS}_{N}$, if two conditions hold: (i) selection opposes $A$ invading $B$, which means that a single mutant of $A$ in a population of $B$ players has a lower fitness; and (ii) selection opposes $A$ replacing $B$, which means $\rho<1 / N$. Therefore, strategy $B$ is $\operatorname{ESS}_{N}$ if

$$
\begin{aligned}
& \text { (i) } b(N-1)<c+d(N-2) \\
& \text { (ii) } a(N-2)+b(2 N-1)<c(N+1)+d(2 N-4)
\end{aligned}
$$

For $N=2$ both conditions reduce to $b<c$. For large populations, the two conditions lead to $b<d$ and $x^{*}>1 / 3$, respectively. Hence, for small populations the traditional ESS concept is neither necessary nor sufficient; for large populations, it is necessary but not sufficient (Fig 3). If we consider a game with many different strategies, then the two conditions must hold in paiwise comparison with every other strategy.

Summing up, (i) in finite populations, natural selection can favor the invasion and replacement of always defect by a reciprocal strategy when starting from a single individual using that strategy. No other mechanism is required. (ii) For any two strategies, natural 
selection can favor $A$ replacing $B$ in a finite population provided $b>c$ or $a-c>2(d-b)$. If $A$ and $B$ are best replies to themselves then the latter condition implies that the frequency of $A$ at the unstable equilibrium, $x^{*}$, must be less than $1 / 3$. (iii) Our analysis leads to natural conditions for evolutionary stability in finite populations. These conditions specify whether a given resident strategy is protected by selection against invasion and replacement of a mutant strategy.

\section{Methods:}

\section{Game dynamics in finite populations}

The fitness of strategies $A$ and $B$ with payoff matrix (1) is, respectively, given by

$$
\begin{aligned}
& f_{i}=1-w+w[a(i-1)+b(N-i)] /[N-1] \\
& g_{i}=1-w+w[c i+d(N-i-1)] /[N-1]
\end{aligned}
$$

Here $i$ denotes the number of individuals using strategy $A$, and $w \in[0,1]$ specifies the contribution of the game to fitness.

Let us calculate the probability, $\rho$, that a single individual $A$ can invade and take over a population of $B$ players. More precisely, $\rho$ is the probability that the stochastic process starting from $i=1$ reaches the absorbing state $i=N$ rather than $i=0$. We obtain ${ }^{24}$

$$
\rho=1 /\left(1+\sum_{k=1}^{N-1} \prod_{i=1}^{k} \frac{g_{i}}{f_{i}}\right)
$$

If $\rho>1 / N$ then selection favors $A$ replacing $B$.

The rate of evolution from all- $A$ to all- $B$ is given by $r=N \rho u$, where $u$ is the mutation rate. We can rescale the rate of evolution in units of $u$. Thus, we set $u=1$. The rate of evolution, $r$ can be an increasing, decreasing or one-humped function of the intensity of selection, $w$, but the following properties are essential for our theorem: (i) $r=1$ if $w=0$ and (ii) if $d r / d w<0$ then $r<1$ for all $w \in(0,1]$.

In the limit of weak selection, $w<<1$, we find that

$$
N \rho \approx 1 /[1-(\alpha N-\beta)(w / 6)]
$$


with $\alpha=a+2 b-c-2 d$ and $\beta=2 a+b+c-4 d$. From this equation, we see that $N \rho>1$ if $\alpha N>\beta$ which leads to (2). If $\alpha>0$ then there is a minimum $N$ for which $N \rho$ can exceed 1. It is given by $N_{\min }=\beta / \alpha$.

\section{TFT and AllD}

Let strategies $A$ and $B$ denote, respectively, TFT and AllD in a Prisoner's Dilemma which is repeated for $n$ rounds on average. The payoff matrix is $a=R n, b=S+P(n-1)$, $c=T+P(n-1)$ and $d=P n$. The parameters of the Prisoner's Dilemma are the reward for mutual cooperation, $R$, the punishment for mutual defection $P$, the sucker's payoff for cooperating with a defector, $S$, and the temptation for defecting against a cooperator, $T$. Since $T>R>P>S$, we have $a>c>d>b$ provided that $n>(T-P) /(R-P)$, which is the minimum number of rounds such that AllD does not dominate TFT.

Condition (2) implies $n(R-P)(N-2)>T(N+1)-S(2 N-1)+P(N-2)$. This determines the minimum number of rounds required for selection to favor TFT replacing AllD for a given population size $N$. We need at least $N=3$. Let $R=3, T=5, P=1$, $S=0$. For $N=3$ we have $n>10.5$. For $N=4$ we have $n>6.75$. For large $N$ we need $n>3$. Similar calculations can be performed for other reciprocal strategies and including errors.

\section{Remarks on ESS}

If $d>b$ then $B$ is both a strict Nash equilibrium and an ESS in comparison with $A$. What is the maximum probability $\rho$ of $A$ replacing $B$ in large populations, $N \rightarrow \infty$ ? We are free to choose $a$ and $c$. In order to maximize $\rho$, we set $a \rightarrow \infty$ and $c=0$. Then we obtain $\rho=[1-w(1-b)] /[2-w(2-b-d)]$. For $w \rightarrow 0$ we have $\rho=1 / 2$. For $w=1$ we have $\rho=b /(b+d)$. Hence there can be enormous selection pressure for replacement of a strict Nash equilibrium even in the limit $N \rightarrow \infty$.

A strict Nash equilibrium implies protection by selection against replacement in the following sense: for a given payoff matrix $(a, b, c, d)$ with $d>b$ and for a given intensity of selection, $w>0$, we have $\rho \rightarrow 0$ as $N \rightarrow \infty$.

For every finite population size, $N$, however, we can calculate the maximum net selective advantage for a mutant replacing a strict Nash equilibrium. Given $b, d$ with 
$d>b$. Let $c=0$ and $w=1$. Calculate $\rho$ for an arbitrarily large, but finite $N$. Let $a \rightarrow \infty$.

We have $\rho \rightarrow b /(b+d)$. This probability of fixation corresponds to a constant relative fitness of $1+(b / d)$ or a net selective advantage of $b / d$.

\section{References}

1. Trivers R, The evolution of reciprocal altruism, Quarterly review of biology 46, 35-57 (1971)

2. Axelrod, R. \& Hamilton, W.D., The evolution of cooperation, Science 211, 1390 (1981).

3. Axelrod, R. The Evolution of Cooperation, (Basic Books, New York, 1984).

4. May R M, More evolution of cooperation, Nature 327, 15-17 (1987)

5. Dugatkin L A Cooperation among animals, OUP 1997

6. Fehr E, Fischbacher U The nature of human altruism, Nature 425, 785-791 (2003)

7. Hammerstein P, Ed, Genetic and cultural evolution of cooperation, MIT Press 2003

8. R. Boyd, H. Gintis, S. Bowles, and P. J. Richerson. The Evolution of Altruistic Punishment. Proc Natl Acad Sci (USA) 100: 35313535, 2003

9. Nowak, M.A. \& Sigmund, K., Tit for tat in heterogeneous populations, Nature 355, 250-253 (1992).

10. Nowak M A, May R M (1992) Evolutionary games and spatial chaos, Nature, 359, 826-829

11. Killingback T, Doebeli M Self-organized criticality in spatial evolutionary game theory, J theor Biol 191: 335-340 (1998)

12. J. Maynard Smith, and G. R Price, Nature 246, 15-18 (1973).

13. P. D. Taylor, and L. Jonker, Math. Biosciences 40, 145-156 (1978)

14. J. Maynard Smith, Evolution and the Theory of Games. (Cambridge University Press, 1982)

15. J. Hofbauer and K. Sigmund, Evolutionary Games and Population Dynamics, (Cambridge University Press, 1998)

16. J. Hofbauer and K. Sigmund, Bull. Amer. Math. Soc. 40, 479-519 (2003).

17. Ficici, S, and J. Pollack [2000]. Effects of Finite Populations on Evolutionary Stable Strategies. Proceedings of the 2000 Genetic and Evolutionary Computation Conference, L. Darrell Whitley (ed.), Morgan-Kaufmann. 
18. Fogel, G. , P. Andrews, and D. Fogel [1998]. "On the instability of evolutionary stable strategies in small populations," Ecological Modeling 109, 283-294.

19. Schaffer, M. [1988]. "Evolutionary Stable Strategies for a Finite Population and a Variable Contest Size," J. Theoretical Biology 132, 469-478.

20. Schreiber, S. [2001]. "Urn Models, Replicator Processes, and Random Genetic Drift." Siam. J. App. Math, 61, 2148-2167.

21. Milinski, M., Tit for tat in sticklebacks and the evolution of cooperation, Nature $\mathbf{3 2 5}$, 433-435 (1987).

22. Moran, P. A. P. (1962) The Statistical Processes of Evolutionary Theory (Clarendon Press, Oxford).

23. Kimura, M. (1968) Nature 217, 624-626.

24. S Karlin \& H M Taylor (1975) A first course in stochastic processes Elsevier

\section{Figure legends}

Fig. 1: Selection can favor the replacement of AllD by TFT in finite populations. (a) The rate of evolution, $N \rho$, is a one humped function of population size $N$. There is an intermediate range of $N$ which leads to positive selection of TFT, $N \rho>1$. (b) $N \rho$ is shown as function of $w$, the intensity of selection. For small $N$, we have $N \rho<1$ for all $w$. For larger $N$ we have $N \rho>1$ for all $w$. For even larger $N$ we have $N \rho>1$ as long as $w$ is below a certain threshold. (c,d) The blue shaded region indicates the parameter region where $N \rho>1$. The light blue line shows the optimum value of $N$ for given $w$ maximizing $N \rho$. The broken red line indicates $N_{\min }=(2 a+b+c-4 d) /(a+2 b-c-2 d)$ which is the predicted minimum population size required for positive selection of TFT in the limit of weak selection. Parameter choices: $R=3, T=5, P=1, S=0 ; n=10$ rounds for (a-c) and $n=4$ rounds for $(\mathrm{d})$.

Fig. 2: (a) The $1 / 3$ law of frequency dependent evolution. Suppose $A$ and $B$ are best replies to themselves, meaning $a>c$ and $d>b$ in payoff matrix (1). In this case all- $A$ and all- $B$ are stable equilibria of the replicator dynamics for infinite population size. The unstable equilibrium is located at a frequency of $A$ given by $x^{*}=(d-b) /(a-b-c+d)$. If $x^{*}<1 / 3$ then $A$ can replace $B$ by positive selection in a sufficiently large, finite population. The minumum population size we need is given by $N_{\min }=(2 a+b+c-4 d) /(a+2 b-c-2 d)$ 
in the case of weak selection $w \rightarrow 0$. (b,c) A strategy is $\mathrm{ESS}_{N}$ if it is protected by selection against invasion and replacement by another strategy for given population size $N$. In the first case, both $A$ and $B$ are clasical ESS, but for $2 \leq N \leq 12$ only $B$ is $\operatorname{ESS}_{N}$, for $12<N<53$ both $A$ and $B$ are $\operatorname{ESS}_{N}$, for $N \geq 53$ only $A$ is $\operatorname{ESS}_{N}$. In the second case, $B$ dominates $A$, but for $2 \leq N \leq 17$ only $A$ is $\operatorname{ESS}_{N}$. For $17<N<22$ both $A$ and $B$ are $\mathrm{ESS}_{N}$. For $N \geq 22$ only $B$ is $\mathrm{ESS}_{N}$. These examples illustrate that for small populations the traditional concept of ESS is neither necessary nor sufficient to imply $\operatorname{ESS}_{N}$ and for large populations it is necessary but not sufficient. 